\title{
Case Report: Type IV Paraesophageal Hernia Repair
}

\author{
Joshua Hefler, $\mathrm{BSc}^{1}$ \\ ${ }^{1}$ Faculty of Medicine, University of Ottawa
}

\section{INTRODUCTION}

Hiatus herniation refers to the transposition of the stomach through the esophageal hiatus of the diaphragm [1]. There are four types of hiatal hernia described [Figure 1]. A type I hernia is also referred to as a sliding hernia, as it may become spontaneously reduced to its normal position. These result from the widening of the crural diaphragm forming the esophageal hiatus and laxity of the phrenoesophageal membrane, causing the transient movement of the gastroesophageal (GE) junction out of the abdomen [1]. Type II hernias are unique and rare - these represent a true paraesopheal herniation of the fundus of the stomach into the chest, with the gastroesophgeal junction being appropriately located [1]. In types III and IV, the gastroesophgeal junction is abnormally positioned in the thorax. They are caused by laxity of the gastrosplenic and gastrocolic ligaments, leading to widening of the esophageal hiatus over time [2]. Type III, or a fixed intrathoracic stomach, is an incarcerated stomach. Type IV hernias are characterized by the presence of other viscerae, such as colon, small bowel or spleen within the hernia sac [2].

Most patients are asymptomatic or may present with reflux [3]. The most common presenting complaint in a patient with a large hiatus hernia is that of anemia - the hernia is found incidentally during endoscopic assessment for other sources of gastrointestinal bleeding [4]. More serious complications, warranting urgent/ emergent surgical management, include gastric volvulus, outlet obstruction, hemorrhage or respiratory distress [2]. With gastric volvulus, patients will experience intermittent obstruction, causing symptoms of dysphagia and regurgitation [2]. Such patients are at increased risk of gastric ulcers, due to delay in gastric emptying and torsion of the stomach wall [2]. Perforation of these ulcers can lead to the emptying of gastric contents into the pleural, pericardial, or mediastinal spaces [2]. Further occult blood loss may occur with obstruction of venous return causing the stomach to become engorged and "weep" blood [2]. In some cases, impairment of circulation may be sufficient to produce necrosis and gangrene [2].
Surgical repair is commonly done laparoscopically, involving reduction of the hernia sac and its contents, followed by closure of the diaphragmatic defect. An esophageal lengthening procedure may be necessary to reduce chance of recurrence [2]. A relaxing diaphragmatic incision and prosthetic mesh reinforcement of the crurae may also be required for adequate closure of the defect, alone or in combination. The repair can also be performed via laparotomy or left thoracotomy.

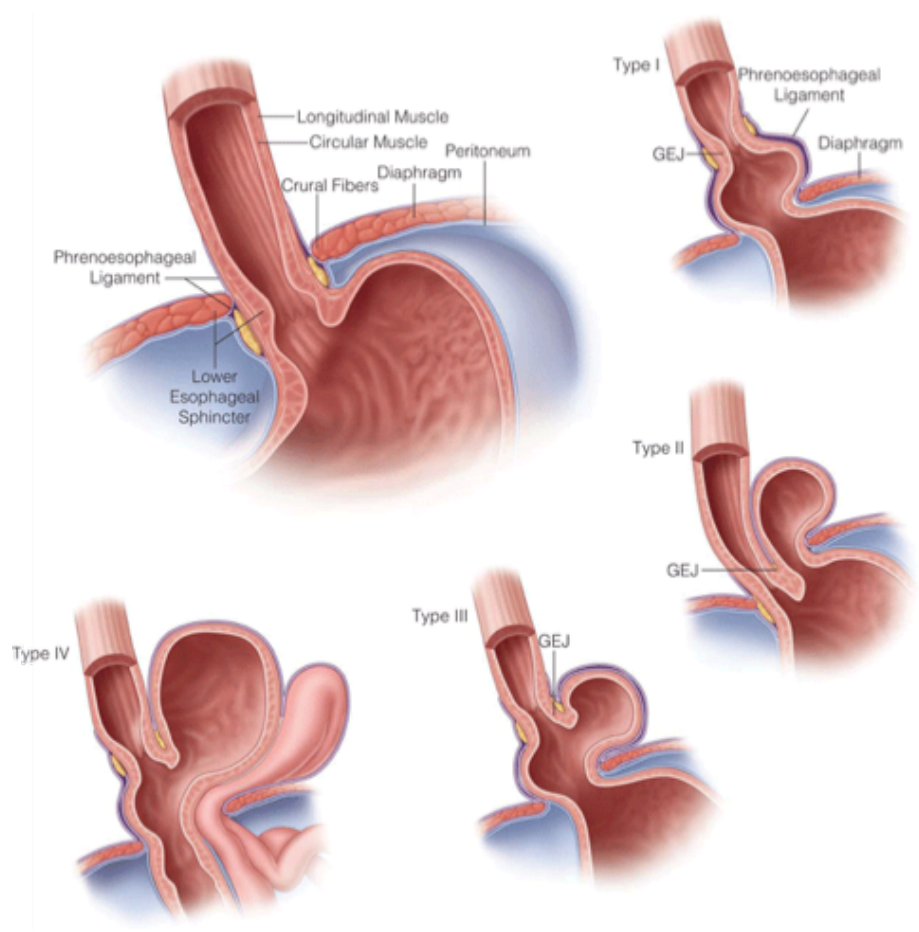

Figure 1. Normal anatomy of the esophageal hiatus shown with examples of different types of hiatal hernias [15].

Keywords: Hiatus Hernia; Hiatal Hernia; Paraesophageal Hernia; Congenital 


\section{CASE}

The patient, a 43-year-old male, presented initially to the emergency department with persistent vomiting and epigastric pain. He was found to have a large incarcerated hiatus hernia. He had no previous complaints of retrosternal discomfort, heartburn, regurgitation or hematemesis. He was managed conservatively with a nasogastric tube decompression and discharged on a liquid diet.

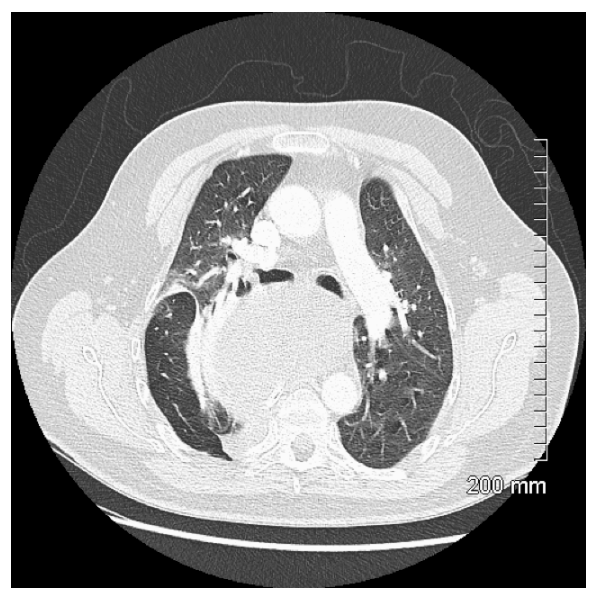

Figure 2. Pre-operative CT Axial View. The heart can be seen in the anterior chest. The left pulmonary artery can be seen towards the right to the image, while the ascending aorta and IVC are on the opposite side. The stomach lies within the thoracic cavity posterior to the heart, with the lesser curvature adjacent to the vertebrae and descending aorta.

His follow up included a CT scan [Figures 1, 2], which demonstrated a type IV paraesophageal hernia. The right-sided diaphragmatic defect measured approximately $16 \mathrm{~cm}$ at the widest point.

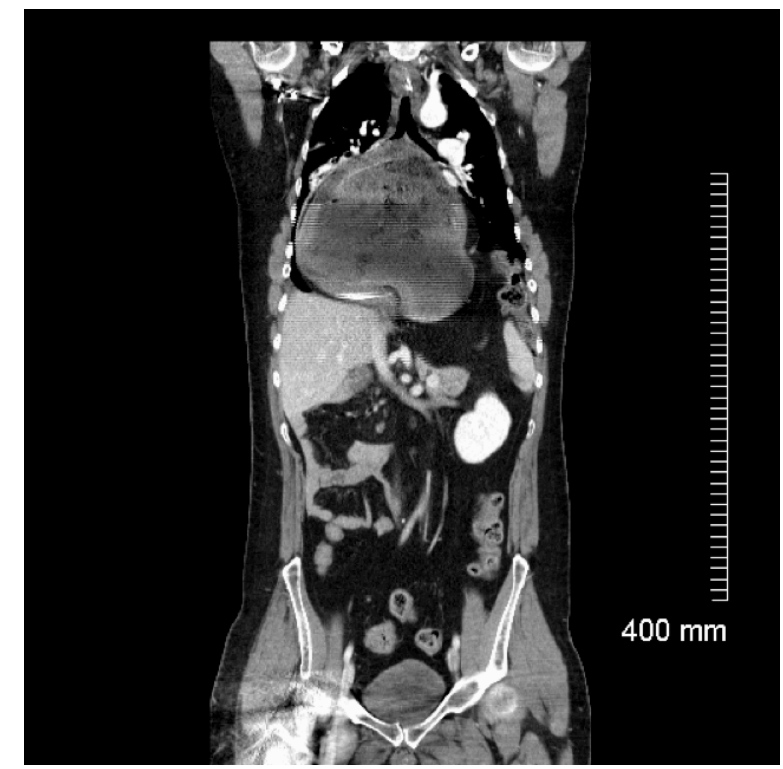

Figure 3. Pre-operative CT Coronal View. Note the large herniation of abdominal contents into the thoracic cavity. The hernia sac sits towards the patient's right side above the liver, adjacent to airspace. The herniation includes the stomach, proximal to the gastric antrum and the small bowel distal to the descending duodenum.
Through this the entire stomach, along with several loops of ileum, the entire right colon, including the appendix and cecum, a portion of the transverse colon, spleen and intra-abdominal fat had herniated into the thoracic cavity. The CT also showed an organoaxial volvulus of the stomach, though not appearing to cause any inflammation or obstruction. A barium study [Figure 4] was also performed.

He was seen in follow up by Thoracic Surgery at the Ottawa Hospital, by which time he was asymptomatic and had resumed his previous diet. The decision was made not to proceed with elective surgery, given the patients absence of symptoms and the lack of obstruction of any of the herniated viscera. However, the patient subsequently presented to the ER approximately 2 months later, with a one-day history of vomiting and epigastric pain, similar to the initial ER visit. He was managed conservatively and discharged after 4 days, but was scheduled for a hernia repair and fundoplication within a month.

The surgical repair proceeded without complication. However, the decision was made to convert from a laparoscopic to open procedure due to lack of adequate exposure. Reduction of several loops of small bowel, as well as the cecum and appendix was achieved before the conversion to an open procedure was made via a midline incision. The unusual position of the cecum in the midline amongst loops of small bowel was taken as evidence for a congenital malrotation. The hernial sac was separated completely from the mediastinum using a combination of harmonic and blunt dissection. Sutures were placed on three fronts to close the hiatus, at its left and right lateral aspects and the posterior aspects of the crura. A bioabsorbable mesh was also used to reinforce the left crus. As an adequate length of

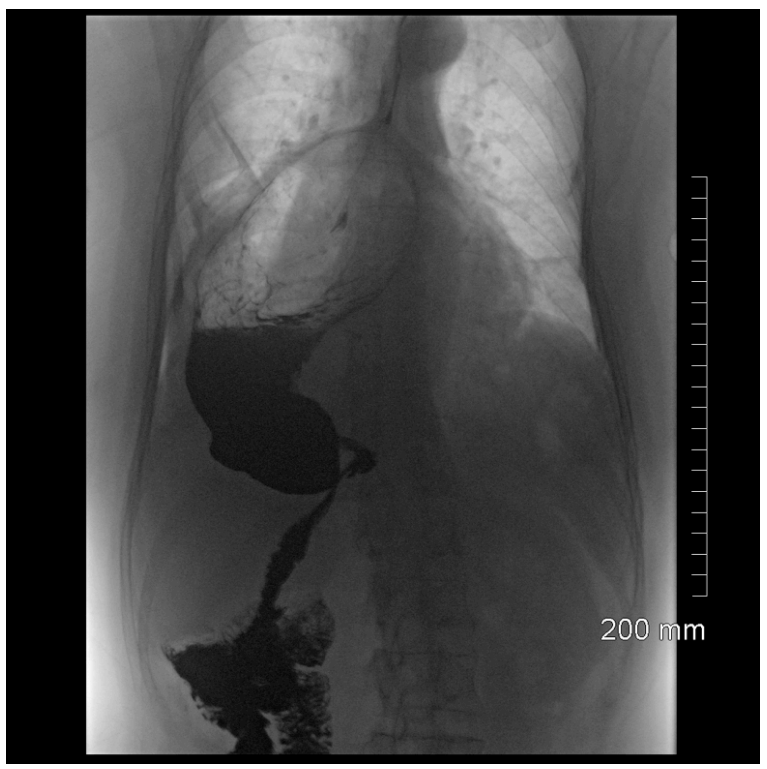

Figure 4. Pre-operative Barium Study. The stomach is seen in the thorax in organoaxial volvulus, causing partial obstruction. Note the barium preparation collecting towards the antrum. 


\section{Case Report}

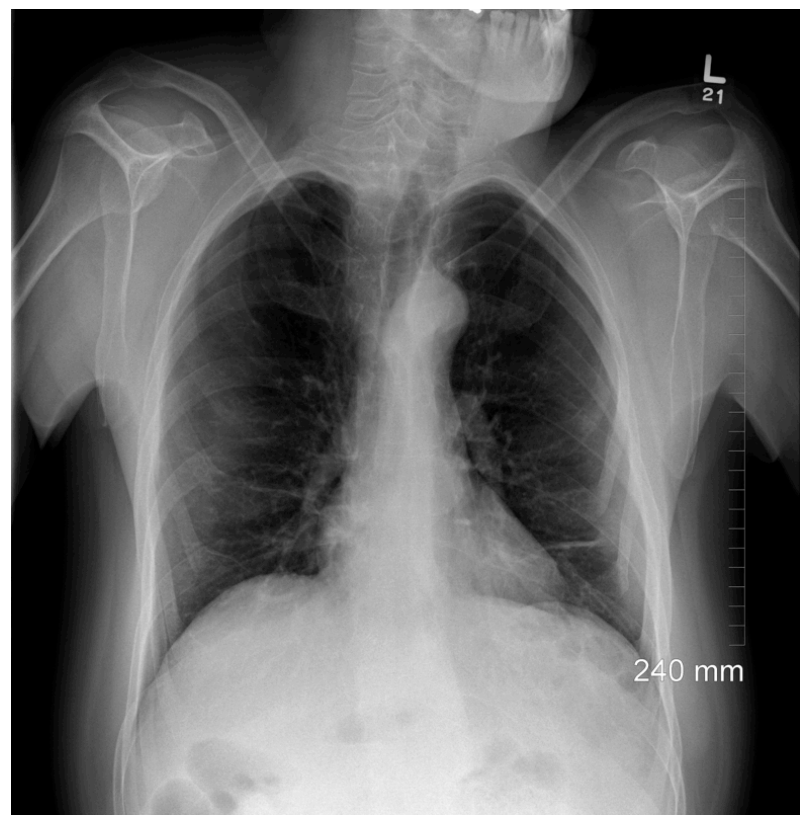

Figure 5. Post-operative Chest X-ray. In contrast to the pre-op imaging, the stomach is no longer in the thorax.

intra-abdominal esophagus was achieved, it was felt unnecessary to perform a fundoplication. However, a gastrostomy tube and sutures were used to straighten the greater curve of the stomach and anchor it to the abdominal wall.

Post-operatively, there were no major complications. A barium study showed no leak in the esophagus. The patient appeared to have gastroparesis (delayed gastric emptying in absence of mechanical obstruction), with the barium being retained in the stomach. A gastrostomy tube was placed as a precaution. This is a well documented complication of gastric and esophageal surgeries, resulting from a stretch injury [5]. This was successfully treated prior to discharge with metoclopramide, which functions to increase gastric motility through antagonism of dopamine receptors in the gut [6]. While the patient did develop wound infection at the site of the gastrostomy tube, no symptomatic or radiological evidence of recurrence was seen six months postoperatively [Figure 5].

\section{DISCUSSION}

Aside from the degree of severity, there are a few other unique aspects to this case that merit comment. Age is a well-known risk factor for hiatus hernia. A review of paraesophageal hernia repairs at the Mayo Clinic reported an average age of 72 at presentation [7]. The relatively young age of the patient suggests that this defect may have at least begun as a congenital deformity. In addition to mild cognitive impairment and the previously mentioned intestinal malrotation, the patient presented with other congenital abnormalities including agenesis of the right kidney and fusion of the posterior elements of T2-T4 vertebrae, both asymptomatic and found incidentally on the pre-operative CT
[Figure 3]. These findings do not fit any classic descriptions of genetic syndromes.

The management of the patient was in line with recommended practices, which consist of initial medical management with surgical repair reserved for urgent/emergent complications or refractory symptoms. The patient presented twice with apparent gastric outlet obstruction, which was twice resolved non-operatively, before the surgery was done electively. In general, surgical correction is recommended in symptomatic patients to prevent serious complications. There was certainly a risk of gastric ischemia in this patient, given the presence of volvulus.

The anatomical presentation and surgical repair are fairly straightforward, but there are some less intuitive points worth mentioning. The patient's stomach was seen in an organoaxial rotation on CT. This is the typical presentation of gastric volvulus in paraesophageal herniation, especially where the GE junction is still fixed to the diaphragm, in which the stomach rotates around the longest axis (from the GE junction to the pylorus) [8]. Mesentericoaxial rotation is less common, occurring around an axis bisecting the lesser and greater curvatures, so that the posterior surface of the stomach ends up facing anteriorly [8].

There are several important surgical considerations. Firstly, the importance of dissecting the hernia sac from the mediastinal structures, which is thought to facilitate intraoperative reduction by releasing the esophagus from any adhesions and has been shown to reduce the risk of early recurrence [9]. Full sac excision is commonly done, as well [9]. One case series has shown decreased early recurrence with sac excision, though it may be technically difficult in the repair of large hernia defects and some concern has been raised about the increased potential for vagal injury, leading to such complications as gastroparesis $[9,10]$. In this case, where the sac was fully excised, the patient's apparent gastroparesis did not persist beyond several days post-operatively, suggesting vagal nerve stretch as a causative factor.

A second point is the importance of closing the diaphragm without tension, especially in the presence of attenuated crura. This attempts to reduce the risk of recurrence via reopening of the defect and is often achieved using a biological mesh, which may be used to cover the defect itself (minimizing tension) or, as in this case, to reinforce to crura $[11,12]$. The use of mesh is recommended for large defects and has been shown to reduce the risk of short-term recurrence [11]. An incision into the diaphragmatic crura may also be necessary to relieve tension [13]. A fundoplication is typically done with a hiatus hernia repair, serving the dual purpose of blocking any transposition of structures through the repaired defect and strengthening the LES to prevent acid reflux [14]. It was not felt to be necessary in this case, given that the length of the esophagus was sufficient and was not likely to have played a role in the initial herniation. However, a gastrostomy tube was used to maintain the position of the stomach and prevent recurrence of volvulus. 
This case details a complicated presentation of a relatively common condition. The anatomical defects are remarkable, given a rather insidious, somewhat non-specific presentation. In addition to being technically interesting, this case highlights some of the important aspects for the surgical management of such patients.

\section{CONSENT}

Oral consent was obtained from both the patient and his caretaker during follow up and is documented in the patient's chart.

\section{REFERENCES}

1. Kahrilas PJ, Kim HC, Pandolfino JE. Approaches to the diagnosis and grading of hiatial hernia. Best Pract Res Clin Gastroenterol. 2008;22(4):601116.

2. Maziak, DE, Pearson, FG. Massive (paraesophageal) hiatial hernia. In: Pearson's Thoracic \& Esophageal Surgery, 3rd ed, Patterson, GA, et al. (Eds); Churchill Livingstone Elsevier, Philedelphia, PA; Vol. 2 2008. 233-238 p.

3. Kahrilas PJ. Hiatus hernia causes reflux?: fact or fiction. Gullet. 1993;3(Suppl):21.

4. Carratt PW, Makar SR, Hong J, et al. Iron-deficiency anemia is a common presenting issue with giant paraesophageal hernia and resolves following repair. J Gastrointest Surg. 2013;17(5):858-862.
5. Patrick A, Epstein O. Review article: gastroparesis. Aliment Pharmacol Ther. 2008;27(9):724-740.

6. Lee A, Kuo B. Metoclopramide in the treatment of diabetic gastroparesis. Expert Rev Endocrinol Metab. 2010;5(5):653-662.

7. Dahlberg PS, Deschamps C, Miller DL, et al. Laparoscopic repair of large paraesophageal hiatial hernia. Ann Thorac Surg. 2001;72(4):1125-1129.

8. Ott DJ, Gelfand DW, Chen YM, et al. Predictive relationship of hiatial hernia to reflux esophagitis. Gastrointest Radiol. 1985;10(4):317.

9. Kohn GP, Price RR, DeMeester DR, et al. Guidelines for the management of hiatal hernia. Surg Endosc. 2013;27:4409-4428.

10. Edye M, Salky B, Posner A, et al. Sac excision is essential to adequate laparoscopic repair of paraesophageal hernia. Surg Endosc. 1998;12:12591263.

11. Schieman C, Grondin SC. Paraesophageal hernia: clinical presentation, evaluation, and management controversies. Thorac Surg Clin. 2009;19:473-484.

12. Antonoff MB, D'Cunha J, Andrade RS, et al. Giant paraesophageal hernia repair: technical pearls. J Thorac Cardiovasc Surg. 2012;144:S67-70.

13. Greene CL, DeMeester SR, Zehetner J, et al. Diaphragmatic relaxing incisions during laparoscopic paraesophageal hernia repair. Surg Endosc. 2013;27(12):4532-4538.

14. Swanstrom LL, Jobe BA, Kinzie LR, et al. Esophageal motility and outcomes following laparoscopic paraesophageal hernia repair and fundoplication. Am J Surg. 1999;177:359-363.

15. Wiener, DC, Wee, JO. Minimally invasive esophageal procedures. In: ACS Surgery: Practice \& Principles, 7th ed, Ashley, S, et al. (Eds); Decker Publishing, Hamilton, ON; Vol. 1 2014. 852 p. 